\title{
Cost Efficient Grooming Based on p-Cycle Protection in Elastic Optical Networks
}

\author{
Jingjing Wu ${ }^{1,}$, Jianfang Zhang ${ }^{1}$ and Yu Du ${ }^{1}$ \\ ${ }^{1}$ P. O. Box 365, School of Computer Science and Engineering, Northeastern University, Shenyang \\ 110819, China \\ awujingjing@cse.neu.edu.cn
}

Keywords: Elastic optical networks, p-Cycle protection, Traffic grooming, Energy efficiency

Abstract: The pre-configured protection cycle, known as p-cycle, is an efficient method, which may overcome long restoration time and complicated protection switching procedure comparing with other schemes. Meanwhile, traffic grooming enabled by sliceable transponders can reduce power consumption and obtain higher spectrum efficiency. In this paper, we study survivable grooming problem based on p-cycle protection in Elastic Optical Networks (EONs). We develop a heuristic algorithm called Shared p-Cycle Grooming Protection (SCGP) to get enough protection and less resources consumption. Numerical simulation results show that the proposed SCGP scheme achieves better performances than the traditional p-Cycle Protection (CP) schemes without grooming.

\section{Introduction}

In recent years, traffic growth results capacity upgrades in telecommunications networks, especially in metro or core networks. As a result, $100 \mathrm{~Gb} / \mathrm{s}$ optical transport systems have been widely deployed, and $400 \mathrm{~Gb} / \mathrm{s}$ and $1 \mathrm{~Tb} / \mathrm{s}$ super-channels have been demonstrated in laboratories and field trials[1]. As a result, the conventional Wavelength Division Multiplexing (WDM) network is challenged when carrying such traffic and applications due to its inflexible grid and coarse bandwidth granularity. In order to make better use of optical resources to accommodate the ever-increasing new internet applications such as Internet Protocol Television (IPTV), video on demand and cloud computing applications, spectrum efficient Elastic Optical Network (EON) based on Orthogonal Frequency Division Multiplexing (OFDM) technology has been proposed as new network architecture [2].

In optical networks, enormous data loss may be experienced in the event of network failures, such as node, link, or channel faults. This problem becomes more compelling when lightpaths are migrated to high bit rates as the EON continues being mature. Therefore, survivability has been a critical issue in the design of the next generation optical networks with very high transmission rate. Some works have extensively studied the survivability in EON [3]. These protection algorithms mentioned above are all based on the path-shared protection mechanism, in which each traffic demand will be first assigned a working path, and then the working path will be assigned a link-disjoint backup path. However, previous works have pointed out that path-shared protection may lead to long restoration time and complicated protection switching procedure. 
In order to solve this problem, previous works have proposed pre-configured protection cycles (p-cycles) [4]. The p-cycle method offers ring-like fast restoration because $p$-cycles are pre-cross-connected. Meanwhile, the p-cycle method offers mesh-like high efficiency. Therefore, p-cycles gather the desired characteristics of mesh-based and ring-based protection methods.

An OFDM-based EON supports various data rates (including sub-wavelength and super-wavelength services) through bandwidth-variable transponders (BVT) at the network edge and bandwidth-variable optical cross-connects (BV-OXCs) in the network core. However, carrying each traffic using an exclusive BV-transponder might not be cost-efficient, especially for sub-wavelength services. Since increasing the number of optical channels increases the optical power injected in optical links, which may not be acceptable for the future development of networks. Traffic grooming is regarded as an effective method for power saving by using fewer transponders and, by avoiding the need of additional guard bands. Survivable traffic grooming[5] that addresses both grooming provisioning and survivability seeks to provide survivable capability for connections and minimize the consumption of spare capacities in the network.

\section{Problem Statement}

The network topology is defined as $G(N, L)$ for an optical mesh network, where $N$ is the set of nodes, and $L$ is the set of fiber links which is bidirectional and contains two unidirectional fibers with contrary direction. In each link an ordered set $F=\left\{f_{1}, f_{2}, \cdots, f_{K}\right\}$ of frequency slots is given. Where $K$ is the maximum number of slots on a link and we assume all links are assigned the same number of slots. Let $R$ denote the set of demands. The $i^{\text {th }}$ demand is denoted as $r_{i}\left(T_{s}, T_{e}, s_{n}\right)$, where $T_{s}$ is the starting node, $T_{e}$ is the ending node, and $s_{n}$ is the number of slots needed by this demand. In fact, there are no starting and ending nodes on a cycle. In order to achieve $p$-cycle protection, a set of $p$-cycles $P C$ is generated based on the physical topology of the network. The $p$-cycle for traffic demand $r_{i}\left(T_{s}, T_{e}, s_{n}\right)$ is denoted as $p c_{i}$. The shortest path algorithm, i.e., Dijkstra's algorithm, is applied to compute the route.

We only illustrate one direction of the p-cycles. An example is given in Fig. 1, there exists two p-cycles 1-0-7-6-5-4-3-2 and 1-0-10-9-8-2. All the links in the network can be divided into two categories: on-cycle links and straddling links. Any single failure of on-cycle link can be protected by the residual links along the $p$-cycle. For example, the failure of link $(0,7)$ can be protected by the backup path 0-1-2-3-4-5-6-7 whose direction is not shown in the figure. On the other hand, any single failure of straddling link can be protected by the residual links along either side. For example, the failure of straddling link $(1,6)$ can be protected by two backup paths 1-0-7-6 and 1-2-3-4-5-6. Now, the two $p$-cycles shares the path 2-1-4. Suppose that a specific sets of contiguous slots (which are highlighted in the figure in red and blue) have been assigned, one guard band is also assigned for each traffic demand. Note that, the free frequency slots besides these occupied slots can be used to accommodate the new traffic demands. Consider the case that there is no grooming, the frequency slot assignment is shown in Fig. 1(b). After traffic grooming, the frequency slot assignment is shown in Fig. 1(c). One can see that the approach in Fig. 1(c) uses fewer transceivers and, by avoiding the need of additional guard band, less frequency slot. 


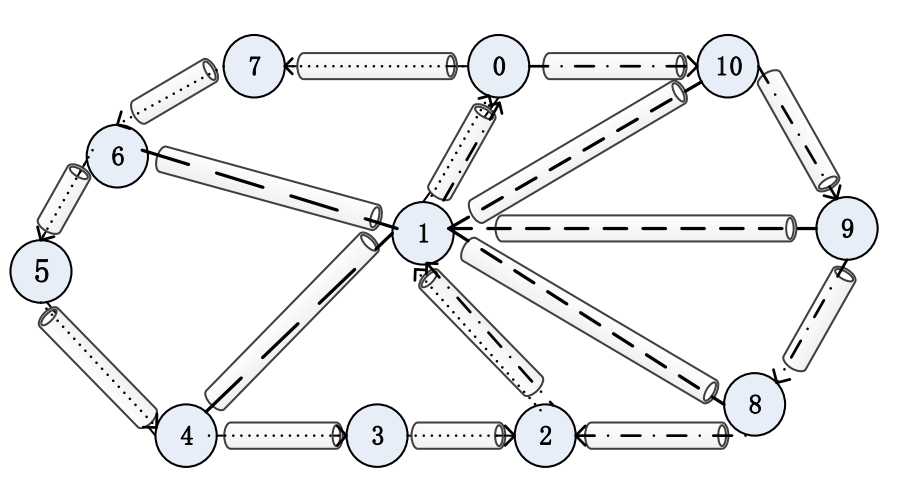

(a)

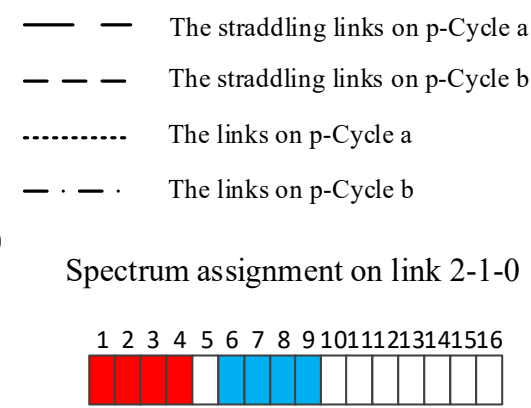

(b)

12345678910111213141516

(c)

Fig. 1. Illustration of grooming $p$-cycles.

\section{Shared $p$-Cycle Grooming Protection (SCGP) Algorithm}

To satisfy a given set of traffic demands, our algorithm sorts these demands and serves each demand one by one. The detailed procedures of SCP are presented as follows.

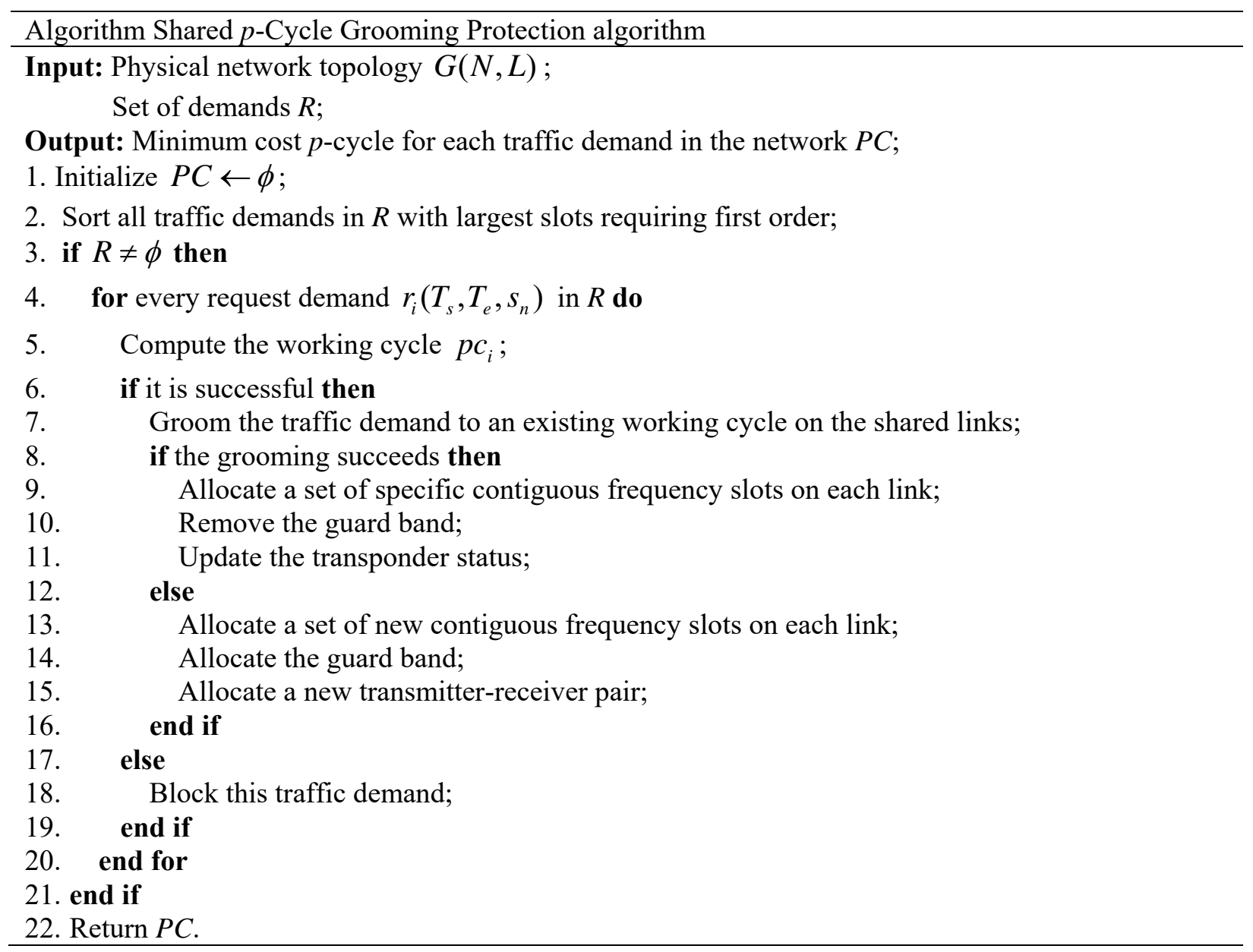




\section{Simulation and analysis}

Now we present the results of the SCGP algorithm. The network is 15 nodes and 27 links that are assumed to be bidirectional fibers. The required bandwidth of each demand varies from 1 slot to 4 slots. In our simulation, we consider the incremental traffic model. In this model, each traffic demand enters the network individually and once allocated, demands in the network cannot be reconfigured. If the resource could not provide service for a traffic demand successfully, the traffic demand will be rejected immediately without waiting in a queue. In simulation, we test the performances of power consumption and number of transponders with and without grooming.

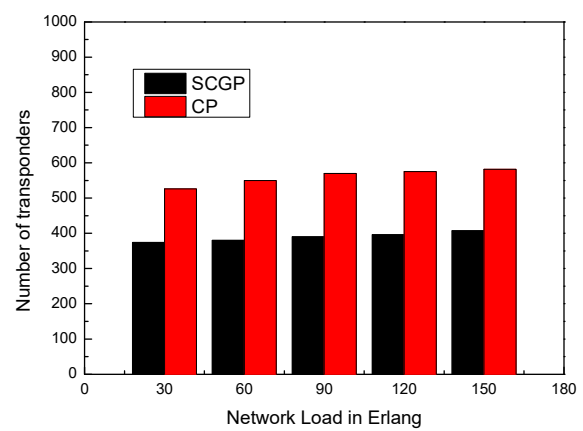

Fig.2. Comparison of number of transponders between SCP and CP with different network load.

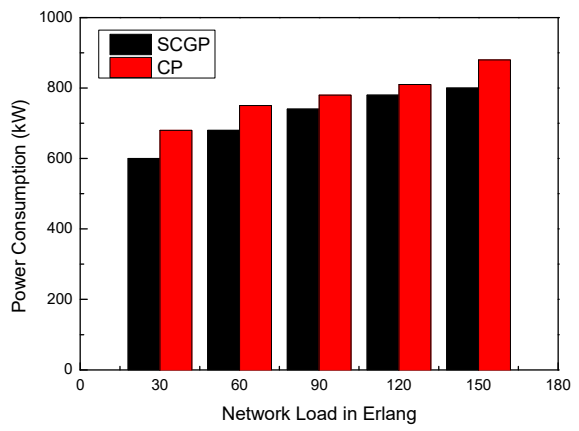

Fig.3. Comparison of power consumption between SCP and CP with different network load.

In Fig. 2, we test the performance of number of transponders for SCGP by comparing with the p-Cycle Protection (CP) without grooming. We assume the network load is from 30 Erlang to 150 Erlang.We can see that the number of transponders for SCGP is lower than that of CP. The reason for this is, due to less consumed transponder resources in SCGP, the utilization ratio of existing lightpath is higher. That means more traffic demands can be grouped together in SCGP. Therefore, the number of new built lightpaths is decreasing and the number of transponders of SCGP is smaller than that of CP.

Fig. 3 shows the power consumption for SCGP by comparing with the CP under different network load. It is shown that with the number of arriving traffic demands becomes larger, the power consumption for SCGP and CP both increase. But we can see that significant power savings are achieved by using grooming technology. 


\section{Acknowledgements}

This work was financially supported by the National Natural Science Foundation of China (61302070, 61501105). The Fundamental Research Funds for the Central Universities (N150404018).

\section{References}

[1] B. Zhu, D. Peckham, A. H. McCurdy, R. Lingle, B. Palsdottir, M. F. Yan, P. W. Wisk, D. J. DiGiovanni: Large-Area Low-Loss Fibers and Advanced Amplifiers for High-Capacity Long-Haul Optical Networks, (Journal of Optical Communications and Networking, 2016).

[2] W. Lu, Z. Zhu: Dynamic service provisioning of advance reservation requests in elastic optical networks, (Journal of Lightwave Technology, 2013).

[3] J. L. Vizcaino, Y. Ye, V. Lopez, F. Jimenez, F. Musumeci: Protection in optical transport networks with fixed and flexible grid: cost and energy efficiency evaluation, (Journal of Optical Switching and Networking, 2014).

[4] M. Ju, F. Zhou, Z. Zhu: Power-efficient protection with directed p-cycles for asymmetric traffic in elastic optical networks, (Journal of Lightwave Technology, 2016).

[5] M. N. Dharmaweera, J. Zhao, L. Yan, M. Karlsson, E. Agrell: Traffic-grooming- and multipath routing-enabled impairment-aware elastic optical networks, (Journal of Optical Communications and Networking, 2016). 